\title{
Isotopism and isomorphism classes of certain Lie algebras over finite fields*
}

\author{
O. J. Falcón ${ }^{1}$, R. M. Falcón ${ }^{2}$ and J. Núñez ${ }^{1}$ \\ ${ }^{1}$ Departamento de Geometría y Topología. \\ Facultad de Matemáticas. Universidad de Sevilla. \\ ${ }^{2}$ Dpto. de Matemática Aplicada I. \\ Universidad de Sevilla. \\ oscfalgan@yahoo.es oscfalgan@us.es jnvaldes@us.es
}

\begin{abstract}
In the current paper, we study the distribution into isotopism and isomorphism classes of the relevant family $\mathcal{F}_{n}^{p}$ of Lie algebras of basis $\left\{e_{1}, \ldots, e_{n}\right\}$ and nonzero brackets $\left[e_{i}, e_{n}\right] \in\left\langle e_{1}, \ldots, e_{n-1}\right\rangle$ over a finite field $\mathbb{F}_{p}$, with $p$ prime. At this end we first introduce the concept of the structure tuple of a Lie algebra and specifically prove that there exist $n$ isotopism classes in $\mathcal{F}_{n}^{p}$ and three families of isomorphism classes depending on the first component of their structure tuple.
\end{abstract}

Keywords: Lie algebra, isotopism, isomorphism.

2010 Mathematics Subject Classification: 17B60, 68W30.

* Preliminary version. The full paper is available in Results in Mathematics DOI: $10.1007 / \mathrm{s} 00025-015-0502-y$

http://link.springer.com/article/10.1007/s00025-015-0502-y 


\section{Introduction.}

An $n$-dimensional Lie algebra $\mathfrak{g}$ over a field $K$ is an $n$-dimensional vector space over $K$ endowed with a second inner law, named bracket product, that is bilinear and anticommutative and that which satisfies the Jacobi identity

$$
J(u, v, w)=[u,[v, w]]+[w,[u, v]]+[v,[w, u]]=0, \text { for all } u, v, w \in \mathfrak{g} .
$$

The center of $\mathfrak{g}$ is the set $Z(\mathfrak{g})=\{u \in \mathfrak{g} \mid[u, v]=0$, for all $v \in \mathfrak{g}\}$, which constitutes an ideal of $\mathfrak{g}$. The Lie algebra is said to be abelian if $Z(\mathfrak{g})=\mathfrak{g}$. Two Lie algebras $\mathfrak{g}$ and $\mathfrak{h}$ are isotopic [1] if there exist three non-singular linear transformations $f, g$ and $h$ from $\mathfrak{g}$ to $\mathfrak{h}$ such that

$$
[f(u), g(v)]=h([u, v]), \text { for all } u, v \in \mathfrak{g} .
$$

Anti-commutativity implies that $f=g$. It is then denoted as $\mathfrak{g} \simeq \mathfrak{h}$ and the tuple $(f, f, h)$ is called an isotopism of Lie algebras. If $h$ is the identity transformation, which will be denoted from now on as $\epsilon$, then the isotopism is said to be principal. On the other hand, if $f=h$, then it is an isomorphism of Lie algebras and it is denoted $f$ instead of $(f, f, f)$. In such a case, it is denoted as $\mathfrak{g} \cong \mathfrak{h}$.

Regarding Lie algebras, it is an interesting topic to consider isotopism and isomorphism classes of finite dimensional Lie algebras over finite fields. This paper addresses both Lie algebras and finite field.

The usual criterion for classifying Lie algebras is that based on isomorphism classes. Nevertheless, the classification of Lie algebras into isotopism classes is also interesting because it allows for combining non-isomorphic algebras that share some properties that are not detected by isomorphisms. The study of classifications of Lie algebras into isotopism classes was recently initiated for filiform Lie algebras in [5]. Previously, isotopisms have also been used to classify distinct algebraic and combinatoric structures such as Jordan algebras [10], alternative algebras [2], division algebras [11], alternating forms [7], quasigroups [6] and Latin squares [8].

Given a Lie algebra of basis $\left\{e_{1}, \ldots, e_{n}\right\}$ and a natural $m \leq n$, let us denote by $V_{m, p}$ the vector space defined over the finite field $\mathbb{F}_{p}$, with $p$ prime, and generated by the elements of the set $\left\{e_{1}, \ldots, e_{m}\right\}$. The current paper addresses the distribution into isomorphism and isotopism classes of the family $\mathcal{F}_{n}^{p}$ of $n$-dimensional Lie algebras defined over $\mathbb{F}_{p}$, such that there exists a basis $\left\{e_{1}, \ldots, e_{n}\right\}$ verifying that

- $\left[e_{i}, e_{j}\right]=0$ for all $i, j<n$.

- $\left[e_{i}, e_{n}\right] \in V_{n-1, p}$.

This family is remarkable because, in a certain sense, it is considered to be the precursor of filiform Lie algebras. Any algebra $\mathfrak{g} \in \mathcal{F}_{n}^{p}$ can be uniquely identified with a structure 
$(n-1)$-tuple $T \in V_{n-1, p}^{n-1}$, whose $i^{t h}$ component $\sum_{j=1}^{n-1} t_{i j} e_{j}$ coincides with $\left[e_{i}, e_{n}\right]$, where $t_{i j} \in \mathbb{F}_{p}$ for all $i, j \in\{1, \ldots, n-1\}$. Such an algebra will be then denoted as $\mathfrak{g}_{T}$. The family $\mathcal{F}_{n}^{p}$ was introduced by Boza et al. [3] for $p=2$, where each algebra of the family was uniquely identified with a directed pseudo-graph and their distribution into isomorphism classes was then explicitly obtained for $n \leq 5$, by considering the properties of such graphs. More recently, the case $p=3$ and $n<5$ was similarly analyzed in [4, 9]. The known isomorphism classes are shown in Table 1, where the representative elements have been chosen to agree with the results of the current paper.

\begin{tabular}{|c|c|c|c|c|}
\hline$p$ & $n$ & Isomorphism classes & & \\
\hline \multirow[t]{19}{*}{2} & 2 & $\mathfrak{h}_{2}^{1}:(0)$ & $\mathfrak{h}_{2}^{2}:\left(e_{1}\right)$ & \\
\hline & 3 & $\mathfrak{h}_{3}^{1}:(0,0)$ & $\mathfrak{h}_{3}^{2}:\left(e_{1}, 0\right)$ & $\mathfrak{h}_{3}^{3}:\left(e_{2}, 0\right)$ \\
\hline & & $\mathfrak{h}_{3}^{4}:\left(e_{1}, e_{2}\right)$ & $h_{3}^{5}:\left(e_{2}, e_{1}\right)$ & $h_{3}^{6}:\left(e_{2}, e_{1}+e_{2}\right)$ \\
\hline & 4 & $\mathfrak{h}_{4}^{1}:(0,0,0)$ & $h_{4}^{2}:\left(e_{1}, 0,0\right)$ & $h_{4}^{3}:\left(e_{2}, 0,0\right)$ \\
\hline & & $\mathfrak{h}_{4}^{4}:\left(e_{1}, e_{2}, 0\right)$ & $h_{4}^{5}:\left(e_{1}, e_{3}, 0\right)$ & $h_{4}^{6}:\left(e_{2}, e_{1}, 0\right)$ \\
\hline & & $\mathfrak{h}_{4}^{7}:\left(e_{2}, e_{1}+e_{2}, 0\right)$ & $\mathfrak{h}_{4}^{8}:\left(e_{2}, e_{3}, 0\right)$ & $\mathfrak{h}_{4}^{9}:\left(e_{1}, e_{2}, e_{3}\right)$ \\
\hline & & $\mathfrak{h}_{4}^{10}:\left(e_{2}, e_{1}, e_{3}\right)$ & $\mathfrak{h}_{4}^{11}:\left(e_{2}, e_{3}, e_{1}\right)$ & $\mathfrak{h}_{4}^{12}:\left(e_{2}, e_{1}+e_{3}, e_{1}\right)$ \\
\hline & & $\mathfrak{h}_{4}^{13}:\left(e_{2}, e_{1}+e_{3}, e_{3}\right)$ & $\mathfrak{h}_{4}^{14}:\left(e_{2}, e_{2}+e_{3}, e_{1}\right)$ & \\
\hline & 5 & $\mathfrak{h}_{5}^{1}:(0,0,0,0)$ & $h_{5}^{2}:\left(e_{1}, 0,0,0\right)$ & $h_{5}^{3}:\left(e_{2}, 0,0,0\right)$ \\
\hline & & $\mathfrak{h}_{5}^{4}:\left(e_{1}, e_{2}, 0,0\right)$ & $\mathfrak{h}_{5}^{5}:\left(e_{1}, e_{3}, 0,0\right)$ & $\mathfrak{h}_{5}^{6}:\left(e_{2}, e_{1}, 0,0\right)$ \\
\hline & & $\mathfrak{h}_{5}^{7}:\left(e_{2}, e_{1}+e_{2}, 0,0\right)$ & $\mathfrak{h}_{5}^{8}:\left(e_{2}, e_{3}, 0,0\right)$ & $\mathfrak{h}_{5}^{9}:\left(e_{3}, e_{4}, 0,0\right)$ \\
\hline & & $\mathfrak{h}_{5}^{10}:\left(e_{1}, e_{2}, e_{3}, 0\right)$ & $\mathfrak{h}_{5}^{11}:\left(e_{1}, e_{2}, e_{4}, 0\right)$ & $\mathfrak{h}_{5}^{12}:\left(e_{2}, e_{1}, e_{3}, 0\right)$ \\
\hline & & $\mathfrak{h}_{5}^{13}:\left(e_{2}, e_{1}, e_{4}, 0\right)$ & $\mathfrak{h}_{5}^{14}:\left(e_{2}, e_{3}, e_{1}, 0\right)$ & $\mathfrak{h}_{5}^{15}:\left(e_{2}, e_{3}, e_{4}, 0\right)$ \\
\hline & & $\mathfrak{h}_{5}^{16}:\left(e_{2}, e_{4}, e_{3}, 0\right)$ & $\mathfrak{h}_{5}^{17}:\left(e_{2}, e_{1}+e_{2}, e_{4}, 0\right)$ & $\mathfrak{h}_{5}^{18}:\left(e_{2}, e_{1}+e_{3}, e_{1}, 0\right)$ \\
\hline & & $\mathfrak{h}_{5}^{19}:\left(e_{2}, e_{1}+e_{3}, e_{3}, 0\right)$ & $\mathfrak{h}_{5}^{20}:\left(e_{2}, e_{2}+e_{3}, e_{1}, 0\right)$ & $\mathfrak{h}_{5}^{21}:\left(e_{1}, e_{2}, e_{3}, e_{4}\right)$ \\
\hline & & $\mathfrak{h}_{5}^{22}:\left(e_{2}, e_{1}, e_{3}, e_{4}\right)$ & $\mathfrak{h}_{5}^{23}:\left(e_{2}, e_{1}, e_{4}, e_{3}\right)$ & $\mathfrak{h}_{5}^{24}:\left(e_{2}, e_{3}, e_{1}, e_{4}\right)$ \\
\hline & & $\mathfrak{h}_{5}^{25}:\left(e_{2}, e_{3}, e_{4}, e_{1}\right)$ & $\mathfrak{h}_{5}^{26}:\left(e_{2}, e_{2}+e_{3}, e_{4}, e_{1}+e_{3}\right)$ & $\mathfrak{h}_{5}^{27}:\left(e_{2}, e_{4}, e_{1}+e_{2}, e_{3}\right)$ \\
\hline & & $\mathfrak{h}_{5}^{28}:\left(e_{2}, e_{2}+e_{4}, e_{1}, e_{3}\right)$ & $\mathfrak{h}_{5}^{29}:\left(e_{2}, e_{2}+e_{3}, e_{1}, e_{4}\right)$ & $\mathfrak{h}_{5}^{30}:\left(e_{2}, e_{3}, e_{1}+e_{2}, e_{4}\right)$ \\
\hline & & $\begin{array}{l}\mathfrak{h}_{5}^{31}:\left(e_{2}, e_{1}+e_{2}, e_{3}+e_{4}, e_{3}\right) \\
\mathfrak{h}_{5}^{34}:\left(e_{2}, e_{3}, e_{4}, e_{1}+e_{3}\right)\end{array}$ & $\mathfrak{h}_{5}^{32}:\left(e_{2}, e_{1}+e_{3}, e_{3}, e_{4}\right)$ & $\mathfrak{h}_{5}^{33}:\left(e_{2}, e_{3}+e_{4}, e_{3}, e_{1}\right)$ \\
\hline \multirow[t]{11}{*}{3} & 2 & $\mathfrak{g}_{2}^{1}:(0)$ & $\mathfrak{g}_{2}^{2}:\left(e_{1}\right)$ & \\
\hline & 3 & $\mathfrak{g}_{3}^{1}:(0,0)$ & $\mathfrak{g}_{3}^{2}:\left(e_{1}, 0\right)$ & $\mathfrak{g}_{3}^{3}:\left(e_{2}, 0\right)$ \\
\hline & & $\mathfrak{g}_{3}^{4}:\left(e_{1}, e_{2}\right)$ & $g_{3}^{5}:\left(e_{2}, e_{1}\right)$ & $g_{3}^{6}:\left(e_{2}, 2 e_{1}\right)$ \\
\hline & & $\mathfrak{g}_{3}^{7}:\left(e_{2}, e_{1}+e_{2}\right)$ & $\mathfrak{g}_{3}^{8}:\left(e_{2}, 2 e_{1}+e_{2}\right)$ & \\
\hline & 4 & $\mathfrak{g}_{4}^{1}:(0,0,0)$ & $g_{4}^{2}:\left(e_{1}, 0,0\right)$ & $g_{4}^{3}:\left(e_{2}, 0,0\right)$ \\
\hline & & $\mathfrak{g}_{4}^{4}:\left(e_{1}, e_{2}, 0\right)$ & $\mathfrak{g}_{4}^{5}:\left(e_{1}, e_{3}, 0\right)$ & $\mathfrak{g}_{4}^{6}:\left(e_{2}, e_{1}, 0\right)$ \\
\hline & & $\mathfrak{g}_{4}^{7}:\left(e_{2}, 2 e_{1}, 0\right)$ & $\mathfrak{g}_{4}^{8}:\left(e_{2}, e_{1}+e_{2}, 0\right)$ & $\mathfrak{g}_{4}^{\overline{9}}:\left(e_{2}, 2 e_{1}+e_{2}, 0\right)$ \\
\hline & & $\mathfrak{g}_{4}^{10}:\left(e_{2}, e_{3}, 0\right)$ & $\mathfrak{g}_{4}^{11}:\left(e_{1}, e_{2}, 2 e_{3}\right)$ & $\mathfrak{g}_{4}^{12}:\left(e_{2}, e_{1}, e_{3}\right)$ \\
\hline & & $\mathfrak{g}_{4}^{13}:\left(e_{2}, 2 e_{1}, e_{3}\right)$ & $\mathfrak{g}_{4}^{14}:\left(e_{2}, e_{1}+e_{2}, e_{3}\right)$ & $\mathfrak{g}_{4}^{15}:\left(e_{3}, e_{2}, e_{1}+e_{2}\right)$ \\
\hline & & $\mathfrak{g}_{4}^{16}:\left(e_{3}, e_{2}, 2 e_{1}+\right.$ & $\mathfrak{g}_{4}^{17}:\left(e_{3}, e_{2}, 2 e_{1}+e_{2}\right.$ & $\mathfrak{g}_{4}^{18}:\left(e_{3}, e_{2}+e_{3}, e_{1}+e_{2}\right)$ \\
\hline & & $\begin{array}{l}\mathfrak{g}_{4}^{19}:\left(e_{3}, e_{2}+e_{3}, e_{1}+e_{2}+e_{3}\right) \\
\mathfrak{g}_{4}^{22}:\left(e_{3}, e_{2}, e_{1}+2 e_{3}\right)\end{array}$ & $\mathfrak{g}_{4}^{20}:\left(e_{3}, e_{2}+e_{3}, e_{1}+2 e_{2}\right)$ & $\mathfrak{g}_{4}^{21}:\left(e_{3}, e_{2}+e_{3}, e_{1}+2 e_{2}+2 e_{3}\right)$ \\
\hline
\end{tabular}

Table 1: Isomorphism classes of $\mathcal{F}_{n}^{p}$.

The current paper addressed from an algebraic point of view, the distribution of $\mathcal{F}_{n}^{p}$ into not only isomorphism classes but also isotopism classes. In Section 2, we prove that there exist $n$ isotopism classes in $\mathcal{F}_{n}^{p}$ whatever $p$ is. Specifically, we obtain that any non-abelian Lie algebra of $\mathcal{F}_{n}^{p}$ is isotopic to that related to a structure tuple $\left(e_{1}, \ldots, e_{m}, 0, \ldots, 0\right) \in$ $V_{n-1, p}^{n-1}$, for some $m \in\{1, \ldots, n-1\}$. Section 3 focuses on the distribution of the set $\mathcal{F}_{n, m}^{p}$ of non-abelian Lie algebras $\mathfrak{g}_{T} \in \mathcal{F}_{n}^{p}$ such that $\operatorname{dim}\left(Z\left(\mathfrak{g}_{T}\right)\right)=n-m-1$. Isomorphism classes are distributed into three families, depending on the first component of their structure tuples. 


\section{Isotopism classes of $\mathcal{F}_{n}^{p}$.}

In the current section, we determine the distribution of $\mathcal{F}_{n}^{p}$ into isotopism classes. We start with a basic property that which must verify any isotopism of Lie algebras.

Lemma 1. Given an isotopism $(f, f, h)$ between two Lie algebras $\mathfrak{g}$ and $\mathfrak{g}^{\prime}$, it is verified that the image by $f$ of the center of $\mathfrak{g}$ coincides with the center of $\mathfrak{g}^{\prime}$.

Proof. Because $f$ is a non-singular linear transformation, we have that $f(\mathfrak{g})=\mathfrak{g}^{\prime}$ and thus, given $u \in Z(\mathfrak{g}),[f(u), v]=h\left(\left[u, f^{-1}(v)\right]\right)=0$ for all $v \in \mathfrak{g}^{\prime}$. Hence, $f(Z(\mathfrak{g})) \subseteq Z\left(\mathfrak{g}^{\prime}\right)$. The equality holds once we consider the isotopism $\left(f^{-1}, f^{-1}, h^{-1}\right)$ between $\mathfrak{g}^{\prime}$ and $\mathfrak{g}$.

The following results focus on the structure tuples of two isotopic Lie algebras of $\mathcal{F}_{n}^{p}$.

Lemma 2. Given two tuples of $V_{n-1, p}^{n-1}$ that are equal up to permutation of their components and relabeling the sub-indices of the elements of $V_{n-1, p}$, the Lie algebras $\mathfrak{g}_{T}$ and $\mathfrak{g}_{T^{\prime}}$ are isotopic.

Proof. Let $T=\left(\sum_{j=1}^{n-1} t_{1 j} e_{j}, \ldots, \sum_{j=1}^{n-1} t_{(n-1) j} e_{j}\right)$ and $T^{\prime}=\left(\sum_{j=1}^{n-1} t_{1 j}^{\prime} e_{j}, \ldots, \sum_{j=1}^{n-1} t_{(n-1) j}^{\prime} e_{j}\right)$ be two tuples of $V_{n-1, p}^{n-1}$ verifying the hypothesis. There exist two permutations $\alpha$ and $\beta$ of the set $\{1, \ldots, n-1\}$ such that $t_{\alpha(i) \beta(j)}^{\prime}=t_{i j}$ for all $i, j \in\{1, \ldots, n-1\}$. It is then sufficient to define, using linearity, the isotopism $(f, f, h)$ such that $f\left(e_{n}\right)=h\left(e_{n}\right)=e_{n}$ and $f\left(e_{i}\right)=e_{\alpha(i)}$ and $h\left(e_{i}\right)=e_{\beta(i)}$ for all $i \in\{1, \ldots, n-1\}$. Here, we have that

$$
\begin{gathered}
{\left[f\left(e_{i}\right), f\left(e_{n}\right)\right]_{\mathfrak{g}_{T^{\prime}}}=\left[e_{\alpha(i)}, e_{n}\right]_{\mathfrak{g}_{T^{\prime}}}=\sum_{j=1}^{n-1} t_{\alpha(i) j}^{\prime} e_{j}=\sum_{j=1}^{n-1} t_{\alpha(i) \beta(j)}^{\prime} e_{\beta(j)}=} \\
=\sum_{j=1}^{n-1} t_{i j} e_{\beta(j)}=h\left(\left[e_{i}, e_{n}\right]_{\mathfrak{g}_{T}}\right),
\end{gathered}
$$

for all $i \in\{1, \ldots, n-1\}$.

Lemma 3. Given $T=\left(t_{1}, \ldots, t_{n-1}\right) \in V_{n-1, p}^{n-1}$ and $i, j \in\{1, \ldots, n-1\}$ such that $i \neq j$, it is verified that $\mathfrak{g}_{T} \simeq \mathfrak{g}_{T^{\prime}}$, where $T^{\prime}=\left(t_{1}, \ldots, t_{i-1}, t_{i}+t_{j}, t_{i+1}, \ldots, t_{n-1}\right) \in V_{n-1, p}^{n-1}$.

Proof. Let us define using linearity the principal isotopism $(f, f, \epsilon)$ such that $f\left(e_{i}\right)=e_{i}-e_{j}$ and $f\left(e_{k}\right)=e_{k}$ for all $k \in\{1, \ldots, n\} \backslash\{i\}$. In particular, we have that

$$
\begin{aligned}
{\left[e_{i}, e_{n}\right]_{\mathfrak{g}_{T^{\prime}}} } & =\left[e_{i}-e_{j}+e_{j}, e_{n}\right]_{\mathfrak{g}_{T^{\prime}}}=\left[f\left(e_{i}+e_{j}\right), f\left(e_{n}\right)\right]_{\mathfrak{g}_{T^{\prime}}}= \\
& =\left[e_{i}+e_{j}, e_{n}\right]_{\mathfrak{g}_{T}}=\left[e_{i}, e_{n}\right]_{\mathfrak{g}_{T}}+\left[e_{j}, e_{n}\right]_{\mathfrak{g}_{T}} .
\end{aligned}
$$


According to Lemma 2 , given any Lie algebra $\mathfrak{g} \in \mathcal{F}_{n}^{p}$, there always exists a structure tuple $T=\left(\sum_{j=1}^{n-1} t_{1 j} e_{j}, \ldots, \sum_{j=1}^{n-1} t_{(n-1) j} e_{j}\right) \in V_{n-1, p}^{n-1}$ such that $\mathfrak{g}_{T} \simeq \mathfrak{g}$ and verifies that, given $i \geq 1$, if $t_{i i}=0, t_{j k}=0$ for all $j, k \geq i$. If $t_{11}=0$, then $\mathfrak{g}$ is the abelian Lie algebra. Otherwise, we define using linearity the isotopism $(\epsilon, \epsilon, h)$ such that $h\left(e_{1}\right)=$ $t_{11}^{p-1}\left(e_{1}-\sum_{j=2}^{n-1} t_{1 j} e_{j}\right)$ and $h\left(e_{j}\right)=e_{j}$ for all $j \neq 1$. We obtain in this way a Lie algebra $\mathfrak{g}_{\bar{T}}$ that is isotopic to $\mathfrak{g}_{T}$ where by

$$
\begin{gathered}
{\left[e_{1}, e_{n}\right]_{\mathfrak{g}_{T}}=\left[\epsilon\left(e_{1}\right), \epsilon\left(e_{n}\right)\right]_{\mathfrak{g}_{T}}=h\left(\left[e_{1}, e_{n}\right]_{\mathfrak{g}_{T}}\right)=h\left(\sum_{j=1}^{n-1} t_{1 j} e_{j}\right)=} \\
t_{11} h\left(e_{1}\right)+\sum_{j=2}^{n-1} t_{1 j} e_{j}=e_{1} .
\end{gathered}
$$

Now, from Lemmas 2 and 3, we can find a structure tuple $T^{\prime}=\left(e_{1}, \sum_{j=2}^{n-1} t_{2 j}^{\prime} e_{j}, \ldots\right.$, $\left.\sum_{j=2}^{n-1} t_{(n-1) j}^{\prime} e_{j}\right) \in V_{n-1, p}^{n-1}$ such that $\mathfrak{g}_{T^{\prime}} \simeq \mathfrak{g}_{\bar{T}} \simeq \mathfrak{g}_{T}$ and such that given $i \geq 2$, if $t_{i i}^{\prime}=0$, then $t_{j k}^{\prime}=0$ for all $j, k \geq i$. If $t_{22}^{\prime}=0$, then $T^{\prime}=\left(e_{1}, 0, \ldots, 0\right)$. Otherwise, we can follow similar reasoning previously used to define $T^{\prime}$ and find a structure tuple $T^{\prime \prime}=$ $\left(e_{1}, e_{2}, \sum_{j=3}^{n-1} t_{3 j} e_{j}, \ldots, \sum_{j=3}^{n-1} t_{(n-1) j} e_{j}\right) \in V_{n-1, p}^{n-1}$ such that $\mathfrak{g}_{T^{\prime \prime}} \simeq \mathfrak{g}_{T^{\prime}}$ and verifies that, given $i \geq 3$, if $t_{i i}=0$, then $t_{j k}=0$ for all $j, k \geq i$. We can repeat the procedure with the remaining components of the structure tuple and hence, the following result holds.

Proposition 1. There exist $n$ isotopism classes in $\mathcal{F}_{n}^{p}$.

Proof. With the previous reasoning, any non-abelian Lie algebra of $\mathcal{F}_{n}^{p}$ is isotopic to an algebra related to a tuple $T_{m}=\left(e_{1}, \ldots, e_{m}, 0, \ldots, 0\right) \in V_{n-1, p}^{n-1}$, for some $m \in\{1, \ldots, n-1\}$. In addition, because $Z\left(\mathfrak{g}_{T_{m}}\right)=\left\langle e_{m+1}, \ldots, e_{n}\right\rangle$ for all $m \in\{1, \ldots, n-1\}$, Lemma 1 implies that $\mathfrak{g}_{T_{m}}$ is not isotopic to $\mathfrak{g}_{T_{l}}$, for any $m, l \in\{1, \ldots, n-1\}$ such that $m \neq l$. Therefore, the $n$-dimensional abelian Lie algebra together with the algebras $\mathfrak{g}_{T_{m}}$ determine the set of isomorphism classes of $\mathcal{F}_{n}^{p}$.

Table 2 shows the distribution into isotopism classes of the isomorphism classes that

\begin{tabular}{|c|c|c|c|c|c|c|}
\hline & $n$ & \multicolumn{3}{|c|}{ Isotopism classes } & & \\
\hline & 2 & $\mathfrak{h}_{2}^{1}$ & $\mathfrak{h}_{2}^{2}$ & & & \multirow{6}{*}{$\mathfrak{h}_{5}^{21} \simeq \ldots \simeq \mathfrak{h}_{5}^{34}$} \\
\hline & 3 & $\mathfrak{h}_{3}^{1}$ & $\mathfrak{h}_{3}^{2} \simeq \mathfrak{h}_{3}^{3}$ & $\mathfrak{h}_{3}^{4} \simeq \mathfrak{h}_{3}^{5} \simeq \mathfrak{h}_{3}^{6}$ & & \\
\hline \multirow{5}{*}{3} & 4 & $\mathfrak{h}_{4}^{1}$ & $\mathfrak{h}_{4}^{2} \simeq \mathfrak{h}_{4}^{3}$ & $\mathfrak{h}_{4}^{4} \simeq \ldots \simeq h_{4}^{8}$ & $\mathfrak{h}_{4}^{9} \simeq \ldots \simeq \mathfrak{h}_{4}^{14}$ & \\
\hline & 5 & $\mathfrak{h}_{5}^{1}$ & $\mathfrak{h}_{5}^{2} \simeq \mathfrak{h}_{5}^{3}$ & $\mathfrak{h}_{5}^{4} \simeq \ldots \simeq \mathfrak{h}_{5}^{9}$ & $\mathfrak{h}_{5}^{10} \simeq \ldots \simeq \mathfrak{h}_{5}^{20}$ & \\
\hline & 2 & $\mathfrak{g}_{2}^{1}$ & & & & \\
\hline & 3 & $\mathfrak{g}_{3}^{1}$ & $\mathfrak{g}_{3}^{2} \simeq \mathfrak{g}_{3}^{3}$ & $\mathfrak{g}_{3}^{4} \simeq \ldots \simeq g_{3}^{8}$ & & \\
\hline & 4 & $\mathfrak{g}_{4}^{1}$ & $\mathfrak{g}_{4}^{2} \simeq \mathfrak{g}_{4}^{3}$ & $\mathfrak{g}_{4}^{4} \simeq \ldots \simeq \mathfrak{g}_{4}^{10}$ & $\mathfrak{g}_{4}^{11} \simeq \ldots \simeq \mathfrak{g}_{4}^{22}$ & \\
\hline
\end{tabular}
appear in Table 1.

Table 2: Isotopism classes of $\mathcal{F}_{n}^{p}$. 


\section{Isomorphism classes of $\mathcal{F}_{n}^{p}$.}

The current section addresses the distribution of $\mathcal{F}_{n}^{p}$ into isomorphism classes. One such class is determined by the abelian Lie algebra. To study the remaining classes, given $m \in$ $\{1, \ldots, n-1\}$, let us denote by $\mathcal{F}_{n, m}^{p}$ the set of non-abelian Lie algebras $\mathfrak{g}_{T} \in \mathcal{F}_{n}^{p}$ such that $\operatorname{dim}\left(Z\left(\mathfrak{g}_{T}\right)\right)=n-m-1$. Because the center of a Lie algebra is preserved by isomorphism, the set $\mathcal{F}_{n, m}^{p}$ is invariant by isomorphism and we can suppose that the structure tuple $T$ of any of its Lie algebras $\mathfrak{g}_{T} \in \mathcal{F}_{n}^{p}$ is of the form $\left(\sum_{j=1}^{n-1} t_{1 j} e_{j}, \ldots, \sum_{j=1}^{n-1} t_{m j} e_{j}, 0, \ldots, 0\right) \in$ $V_{n-1, p}^{n-1}$, where the rows of the coefficient matrix $\left(t_{i j}\right)$ are linearly independent. Otherwise, it is sufficient to consider a change of the basis of $\mathfrak{g}_{T}$ to obtain that $t_{i j}=0$ for all $i>m$ and $j<n$. The following results are satisfied

Lemma 4. Given a Lie algebra $\mathfrak{g}_{T} \in \mathcal{F}_{n, m}^{p}$, it is verified that $\mathfrak{g}_{T} \cong \mathfrak{g}_{k T}$ for all $k \in \mathbb{F}_{p} \backslash\{0\}$.

Proof. It is sufficient to define using linearity the isomorphism $f$ such that $f\left(e_{i}\right)=k e_{i}$ if $i<n$ and $f\left(e_{n}\right)=e_{n} / k$, because we then obtain

$$
\left[e_{i}, e_{n}\right]_{\mathfrak{g}_{k T}}=\left[k e_{i}, e_{n} / k\right]_{\mathfrak{g}_{k T}}=\left[f\left(e_{i}\right), f\left(e_{n}\right)\right]_{\mathfrak{g}_{k T}}=f\left(\left[e_{i}, e_{n}\right]_{\mathfrak{g}_{T}}\right)=\sum_{j=1}^{n-1} k t_{i j} e_{j} .
$$

Lemma 5. Given a tuple $T=\left(\sum_{j=1}^{n-1} t_{1 j} e_{j}, \ldots, \sum_{j=1}^{n-1} t_{m j} e_{j}, 0, \ldots, 0\right) \in V_{n-1, p}^{n-1}$ such that there exists a natural $i \leq m$ so that $t_{i i} \neq 0$, the Lie algebra $\mathfrak{g}_{T} \in \mathcal{F}_{n, m}^{p}$ is isomorphic to the Lie algebra $\mathfrak{h} \in \mathcal{F}_{n, m}^{p}$, where

$$
\left[e_{j}, e_{n}\right]_{\mathfrak{h}}=\left\{\begin{array}{l}
\sum_{k \leq m} t_{i k} e_{k}, \text { if } j=i \\
{\left[e_{j}, e_{n}\right]_{\mathfrak{g}_{T}}, \text { otherwise }}
\end{array}\right.
$$

Proof. It is sufficient to define using linearity the isomorphism $f$ such that $f\left(e_{i}\right)=e_{i}-$ $t_{i i}^{p-1} \sum_{j>m} t_{i j} e_{j}$ and $f\left(e_{j}\right)=e_{j}$ for all $j \neq i$. The Lie algebra $\mathfrak{h} \in \mathcal{F}_{n, m}^{p}$ isomorphic to $\mathfrak{g}$ with respect to $f$ verifies that

$$
\begin{gathered}
{\left[e_{i}, e_{n}\right]_{\mathfrak{h}}=\left[f\left(e_{i}+t_{i i}^{p-1} \sum_{j>m} t_{i j} e_{j}\right), f\left(e_{n}\right)\right]_{\mathfrak{h}}=f\left(\left[e_{i}+t_{i i}^{p-1} \sum_{j>m} t_{i j} e_{j}, e_{n}\right]_{\mathfrak{g}_{T}}\right)=} \\
=f\left(\left[e_{i}, e_{n}\right]_{\mathfrak{g}_{T}}\right)=\sum_{j \neq i} t_{i j} e_{j}+t_{i i} f\left(e_{i}\right)=\sum_{j \leq m} t_{i j} e_{j} .
\end{gathered}
$$

Given a Lie algebra $\mathfrak{g} \in \mathcal{F}_{n, m}^{p}$ and a natural $i \leq m$, we consider the number $\rho_{\mathfrak{g}, i}=$ $\min \left\{j<n: t_{i j} \neq 0\right\}$. The following results hold 
Lemma 6. Any Lie algebra $\mathfrak{g} \in \mathcal{F}_{n, m}^{p}$ is isomorphic to a Lie algebra $\mathfrak{g}_{T} \in \mathcal{F}_{n, m}^{p}$ of structure tuple $T=\left(\sum_{j=1}^{n-1} t_{1 j} e_{j}, \ldots, \sum_{j=1}^{n-1} t_{m j} e_{j}, 0, \ldots, 0\right) \in V_{n-1, p}^{n-1}$ such that $t_{i \rho_{\mathfrak{g}, j}}=0$ for all $i, j \leq m$ such that $i \neq j$.

Proof. We define using linearity the isomorphism $f_{1}$ such that $f\left(e_{1}\right)=e_{1}$ and $f\left(e_{i}\right)=$ $e_{i}-t_{1 \rho_{\mathfrak{g}, 1}}^{p-1} t_{i \rho_{\mathfrak{g}, 1}} e_{1}$ for all $i>1$. The only nonzero coefficient of $e_{\rho_{\mathfrak{g}, 1}}$ in the structure tuple of the Lie algebra $\mathfrak{g}_{1} \in \mathcal{F}_{n, m}^{p}$ isomorphic to $\mathfrak{g}$ with respect to $f_{1}$ is that of the first component of such a tuple.

Proposition 2. Given a Lie algebra $\mathfrak{g}_{T} \in \mathcal{F}_{n, m}^{p}$ of the structure tuple $T=\left(\sum_{j=1}^{n-1} t_{1 j} e_{j}, \ldots\right.$, $\left.\sum_{j=1}^{n-1} t_{m j} e_{j}, 0, \ldots, 0\right) \in V_{n-1, p}^{n-1}$, is isomorphic to a Lie algebra $\mathfrak{h} \in \mathcal{F}_{n, m}^{p}$ verifying that

$$
\left[e_{1}, e_{n}\right]_{\mathfrak{h}}=\left\{\begin{array}{l}
e_{1}, \text { if }\left[e_{1}, e_{n}\right]_{\mathfrak{g}_{T}}=t_{11} e_{1}, \\
e_{2}, \text { if there exists } j \in\{2, \ldots, m\} \text { such that } t_{1 j} \neq 0, \\
e_{m+1}, \text { if } j_{1}>m
\end{array}\right.
$$

Proof. We can suppose that $t_{1 j_{1}}=1$. Otherwise, we use the Lie algebra $\mathfrak{g}_{t_{1 j_{1}}^{p-1} T}$, which is isomorphic to $\mathfrak{g}_{T}$ from Lemma 4 . We study each case separately.

- If $\left[e_{1}, e_{n}\right]_{\mathfrak{g}_{T}}=e_{1}$, then the result follows in a straightforward manner by considering $\mathfrak{h}=\mathfrak{g}_{T}$.

- If $j_{1}=1$ and if there exists $j>1$ such that $t_{1 j} \neq 0$, we can then suppose from Lemma 6 that $j \leq m$. It is then sufficient to define using linearity the isomorphism $f$ such that $f\left(e_{i}\right)=e_{i}$ if $i \neq j$ and $f\left(e_{j}\right)=t_{1 j}^{p-1}\left(e_{j}-e_{1}-\sum_{i \notin\{1, j\}} t_{1 i} e_{i}\right)$. The Lie algebra $\mathfrak{g}^{\prime}$ isomorphic to $\mathfrak{g}_{T}$ with respect to $f$ verifies that

$$
\left[e_{1}, e_{n}\right]_{\mathfrak{g}^{\prime}}=\left[f\left(e_{1}\right), f\left(e_{n}\right)\right]_{\mathfrak{g}^{\prime}}=f\left(\left[e_{1}, e_{n}\right]_{\mathfrak{g}_{T}}\right)=e_{j} .
$$

Now, we can define using linearity the isomorphism $g$ such that $g\left(e_{j}\right)=e_{2}, g\left(e_{2}\right)=e_{j}$ and $g\left(e_{i}\right)=e_{i}$ for all $i \notin\{2, j\}$. The Lie algebra $\mathfrak{h}$ isomorphic to $\mathfrak{g}^{\prime}$ with respect to $g$ verifies that $\left[e_{1}, e_{n}\right]_{\mathfrak{h}}=e_{2}$.

- Finally, if $j_{1} \neq 1$, then we define using linearity the isomorphism $f$ such that $f\left(e_{i}\right)=$ $e_{i}$ if $i \neq j_{1}$ and that $f\left(e_{j_{1}}\right)=e_{j_{1}}-\sum_{j>j_{1}} t_{1 j} e_{j}$. The Lie algebra $\mathfrak{g}^{\prime}$ isomorphic to $\mathfrak{g}_{T}$ with respect to $f$ verifies that

$$
\left[e_{1}, e_{n}\right]_{\mathfrak{g}^{\prime}}=\left[f\left(e_{1}\right), f\left(e_{n}\right)\right]_{\mathfrak{g}^{\prime}}=f\left(\left[e_{1}, e_{n}\right]_{\mathfrak{g}_{T}}\right)=f\left(e_{j_{1}}\right)+\sum_{j>j_{1}} t_{1 j} e_{j}=e_{j_{1}} .
$$

If $j_{1} \in\{2, \ldots, m\}$, we can then use the isomorphism $g$ defined in the previous case to obtain a Lie algebra $\mathfrak{h}$ isomorphic to $\mathfrak{g}_{T}$ and such that $\left[e_{1}, e_{n}\right]_{\mathfrak{h}}=e_{2}$. Analogously, if $j \geq m$, then it is sufficient to define using linearity the isomorphism $g$ such that $g\left(e_{j}\right)=e_{m}, g\left(e_{m}\right)=e_{j}$ and $g\left(e_{i}\right)=e_{i}$ for all $i \notin\{m, j\}$. 
The previous result can be used to determine the distribution of $\mathcal{F}_{n, 1}^{p}$ into isomorphism classes.

Theorem 1. Given $n>2$ and $p$ prime, there exist two isomorphism classes of Lie algebras of $\mathcal{F}_{n, 1}^{p}$. They are determined by the structure tuples $\left(e_{1}, 0, \ldots, 0\right)$ and $\left(e_{2}, 0, \ldots, 0\right)$ of $V_{n-1, p}^{n-1}$

Proof. According to Proposition 2, any Lie algebra of $\mathcal{F}_{n, 1}^{p}$ is isomorphic to $\mathfrak{g}_{\left(e_{1}, 0, \ldots, 0\right)}$ or $\mathfrak{g}_{\left(e_{2}, 0, \ldots, 0\right)}$ in $\mathcal{F}_{n, 1}^{p}$. Moreover, $\mathfrak{g}_{1}$ and $\mathfrak{g}_{2}$ are not isomorphic because isomorphisms preserve the center of Lie algebras and, in our case, $\left[\mathfrak{g}_{2}, \mathfrak{g}_{2}\right] \in Z\left(\mathfrak{g}_{2}\right)$, but $\left[\mathfrak{g}_{1}, \mathfrak{g}_{1}\right] \notin Z\left(\mathfrak{g}_{1}\right)$.

Even if Proposition 2 is useful for simplifying the first component of the structure tuples of the representative Lie algebras of the isomorphism classes of $\mathcal{F}_{n, m}^{p}$, it cannot be directly used to classify them. One can observe, for instance, that the Lie algebras $\mathfrak{g}_{\left(e_{1}, e_{3}, 0\right)}$ and $\mathfrak{g}_{\left(e_{2}, e_{2}+e_{3}, 0\right)}$ in $\mathcal{F}_{n, 2}^{2}$ are isomorphic, but their structure tuples have distinct first components in $\left\{e_{1}, e_{2}\right\}$. Indeed, the majority of the isomorphism classes of $\mathcal{F}_{n, m}^{p}$ can be rewritten in such a way that the first component of their structure tuples is $e_{2}$. To prove this, the following result determines explicitly the isomorphism classes of $\mathcal{F}_{n, m}^{p}$ that do not have any representative Lie algebra with a structure tuple of the first component equal to $e_{2}$. It is the case, for instance, for the isomorphism class $\mathfrak{h}_{5}^{7}$ of structure tuple $\left(e_{1}, e_{3}, 0,0\right)$ in Table 1.

Theorem 2. Given a Lie algebra $\mathfrak{g}_{T} \in \mathcal{F}_{n, m}^{p}$, if $\mathfrak{g}_{T}$ is not isomorphic to a Lie algebra $\mathfrak{h} \in \mathcal{F}_{n, m}^{p}$ such that $\left[e_{1}, e_{n}\right]_{\mathfrak{h}}=e_{2}$, then there exists $m^{\prime} \in\{0, \ldots, m\}$ such that $2 m-m^{\prime} \leq n-1$ and $\mathfrak{g}_{T}$ is isomorphic to a Lie algebra of structure tuple of the form $\left(e_{1}, t_{2} e_{2} \ldots, t_{m^{\prime}} e_{m^{\prime}}, e_{m+1}, \ldots, e_{2 m-m^{\prime}}, 0, \ldots, 0\right) \in V_{n-1, p}^{n-1}$, where $t_{2} \leq \ldots \leq t_{m^{\prime}}$.

Proof. If $T=\left(\sum_{j=1}^{n-1} t_{1 j} e_{j}, \ldots, \sum_{j=1}^{n-1} t_{m j} e_{j}, 0, \ldots, 0\right) \in V_{n-1, p}^{n-1}$ is the structure tuple of $\mathfrak{g}_{T}$, then the hypothesis is possible only if $t_{i j}=0$ for all $i, j \leq m$ such that $i \neq j$. Otherwise, we can define using using linearity an isomorphism $f$ such that $f\left(e_{1}\right)=e_{i}, f\left(e_{i}\right)=e_{1}$ and $f\left(e_{k}\right)=e_{k}$ if $k \notin\{1, i\}$. The Lie algebra $\mathfrak{h} \in \mathcal{F}_{n, m}^{p}$ isomorphic to $\mathfrak{g}_{T}$ with respect to $f$ should then verify that the product $\left[e_{1}, e_{n}\right]_{\mathfrak{h}}$ would contain the addend $t_{i j} e_{j}$, with $t_{i j} \neq 0$, and Proposition 2 would imply that $\mathfrak{h}$ is isomorphic to a Lie algebra $\mathfrak{h}^{\prime}$ such that $\left[e_{1}, e_{n}\right]_{\mathfrak{h}^{\prime}}=e_{2}$. Hence, it must be

$$
\left[e_{i}, e_{n}\right]_{\mathfrak{g}_{T}}=t_{i i} e_{i}+\sum_{j=m+1}^{n-1} t_{i j} e_{j}, \text { for all } i \leq m .
$$

Let $m^{\prime}$ be the number of naturals $i \in\{1, \ldots, m\}$ such that $t_{i i} \neq 0$ and let us define using linearity the isomorphism $f$ such that $f\left(e_{i}\right)=t_{i i}^{p-1}\left(e_{i}-\sum_{j=m+1}^{n-1} t_{i j} e_{j}\right)$, if $t_{i i} \neq 0$; otherwise, 
$f\left(e_{i}\right)=e_{i}$. The Lie algebra $\mathfrak{h}$ isomorphic to $\mathfrak{g}_{T}$ with respect to $f$ verifies that

$$
\left[e_{i}, e_{n}\right]_{\mathfrak{h}}=\left\{\begin{array}{l}
t_{i i} e_{i}, \text { if } t_{i i} \neq 0, \\
\sum_{j=m+1}^{n-1} t_{i j} e_{j}, \text { otherwise. }
\end{array}\right.
$$

Because $\mathfrak{h} \in \mathcal{F}_{n, m}^{p}$, there exists $\left(m-m^{\prime}\right)$ distinct naturals $i_{1}, \ldots, i_{m-m^{\prime}} \leq m$ and $\left(m-m^{\prime}\right)$ distinct naturals $j_{1}, \ldots, j_{m-m^{\prime}} \in\{m+1, \ldots, n-1\}$ such that $t_{i_{k} j_{k}} \neq 0$ for all $k \leq m-m^{\prime}$. Let us define using linearity the isomorphism $g$ such that

$$
g\left(e_{i}\right)=\left\{\begin{array}{l}
e_{i}, \text { if } i \notin\left\{i_{1}, \ldots, i_{m-m^{\prime}}\right\}, \\
t_{i_{k} j_{k}}{ }^{p-1}\left(e_{j_{k}}-\sum_{j \neq j_{k}} t_{i_{k} j} e_{j}\right), \text { if } i=i_{k} \text { for some } k \leq m-m^{\prime} .
\end{array}\right.
$$

After applying the isomorphism $g$ to $\mathfrak{h}$, we can relabel conveniently the elements of the basis and apply Lemma 4 to find $m-1$ numbers $t_{2}, \ldots, t_{m^{\prime}}, t_{m+1}, \ldots, t_{2 m-2} \in \mathbb{F}_{p} \backslash\{0\}$, such that $t_{2} \leq \ldots \leq t_{m^{\prime}}$ and the Lie algebra $\mathfrak{g}_{T^{\prime}}$ with $T^{\prime}=\left(e_{1}, t_{2} e_{2}, \ldots, t_{m^{\prime}} e_{m^{\prime}}, t_{m+1} e_{m+1}\right.$, $\left.\ldots, t_{2 m-m^{\prime}} e_{2 m-m^{\prime}}, 0, \ldots, 0\right) \in V_{n-1, p}^{n-1}$ structure tuple is isomorphic to $\mathfrak{h}$. In particular, it must be $2 m-m^{\prime} \leq n-1$. Finally, if we define using linearity the isomorphism $h$ such that $h\left(e_{i}\right)=t_{i}^{p-1} e_{i}$ if $i \in\left\{m+1, \ldots, 2 m-m^{\prime}\right\}$, and $h\left(e_{i}\right)=e_{i}$, otherwise, then the Lie algebra $\mathfrak{g}_{T^{\prime}}$ is isomorphic to that of structure tuple $\left(e_{1}, t_{2} e_{2}, \ldots, t_{m^{\prime}} e_{m^{\prime}}, e_{m+1}, \ldots, e_{2 m-m^{\prime}}, 0, \ldots\right.$, $0) \in V_{n-1, p}^{n-1}$.

Once we have identified the isomorphism classes of $\mathcal{F}_{n, m}^{p}$ of Theorem 2 , we focus our study on those Lie algebras $\mathfrak{g}_{T} \in \mathcal{F}_{n, m}^{p}$ of structure tuple $T=\left(e_{2}, \sum_{j=1}^{n-1} t_{2 j} e_{j}, \ldots\right.$, $\left.\sum_{j=1}^{n-1} t_{m j} e_{j}, 0, \ldots, 0\right) \in V_{n-1, p}^{n-1}$. We can suppose that $t_{i 2}=0$ for all $i>2$. Otherwise, we define using linearity the isomorphism $f$ such that $f\left(e_{j}\right)=e_{j}$ if $j \neq i$ and $f\left(e_{i}\right)=e_{i}+t_{i 2} e_{1}$. Let us consider the number $j_{2}=\min \left\{j<n: j \neq 2\right.$ and $\left.t_{2 j} \neq 0\right\}$. Analogously to Proposition 2 and Theorem 2, the next result holds

Proposition 3. Given a Lie algebra $\mathfrak{g}_{T} \in \mathcal{F}_{n, m}^{p}$ of structure tuple $T=\left(e_{2}, \sum_{j=1}^{n-1} t_{2 j} e_{j}, \ldots\right.$, $\left.\sum_{j=1}^{n-1} t_{m j} e_{j}, 0, \ldots, 0\right) \in V_{n-1, p}^{n-1}$, it is isomorphic to a Lie algebra $\mathfrak{h} \in \mathcal{F}_{n, m}^{p}$ verifying that $\left[e_{1}, e_{n}\right]_{\mathfrak{h}}=e_{2}$ and

$$
\left[e_{2}, e_{n}\right]_{\mathfrak{h}}=\left\{\begin{array}{l}
t_{21} e_{1}+t_{22} e_{2}, \text { if } j_{2}=1, \\
t_{22} e_{2}+e_{3}, \text { if } j_{2} \in\{3, \ldots, m\}, \\
t_{22} e_{2}+e_{m+1}, \text { if } j_{2}>m .
\end{array}\right.
$$

In particular, if $\mathfrak{g}_{T}$ is not isomorphic to a Lie algebra $\mathfrak{h} \in \mathcal{F}_{n, m}^{p}$ such that $\left[e_{2}, e_{n}\right]_{\mathfrak{h}} \in$ $\left\langle e_{1}, e_{2}, e_{3}\right\rangle$, then it is isomorphic to the Lie algebra of structure tuple $\left(e_{2}, t_{22} e_{2}+e_{m+1}, \ldots\right.$, $\left.e_{2 m-1}, 0, \ldots, 0\right) \in V_{n-1, p}^{n-1}$.

We study each one of the three cases of Proposition 3 . 
Similar to what is observed for $\left[e_{1}, e_{n}\right]$, the majority of the isomorphism classes of $\mathcal{F}_{n, m}^{p}$ can be rewritten in such a way that the first components of their structure tuples are $e_{2}$.

To this end, we invoke a previous definition. Given a natural $m^{\prime}<n-m$ and two Lie algebras $\mathfrak{g}_{T} \in \mathcal{F}_{n, m^{\prime}}^{p}$ and $\mathfrak{g}_{T^{\prime}} \in \mathcal{F}_{n-m^{\prime}, m-m^{\prime}}^{p}$ of respective structure tuples

$$
T=\left(\sum_{j=1}^{n-1} t_{1 j} e_{j}, \ldots, \sum_{j=1}^{n-1} t_{m^{\prime} j} e_{j}, 0, \ldots, 0\right) \in V_{n-1, p}^{n-1}
$$

and

$$
T^{\prime}=\left(\sum_{j=1}^{n-m^{\prime}-1} t_{1 j}^{\prime} e_{j}, \ldots, \sum_{j=1}^{n-m^{\prime}-1} t_{\left(m-m^{\prime}\right) j}^{\prime} e_{j}, 0, \ldots, 0\right) \in V_{n-m^{\prime}-1, p}^{n-m^{\prime}-1},
$$

we define the Lie algebra $\mathfrak{g}_{T \oplus T^{\prime}} \in \mathcal{F}_{n, m}^{p}$ of structure tuple

$$
T \oplus T^{\prime}=\left(\sum_{j=1}^{n-1} t_{1 j} e_{j}, \ldots, \sum_{j=1}^{n-1} t_{m^{\prime} j} e_{j}, \sum_{j=1}^{n-m^{\prime}-1} t_{1 j}^{\prime} e_{m^{\prime}+j}, \ldots, \sum_{j=1}^{n-m^{\prime}-1} t_{m^{\prime} j}^{\prime} e_{m^{\prime}+j}, 0, \ldots, 0\right) .
$$

The following result holds

Theorem 3. Given a Lie algebra $\mathfrak{g} \in \mathcal{F}_{n, m}^{p, 1}$, there exists a Lie algebra $\mathfrak{g}^{\prime} \in \mathcal{F}_{n-1, m-1}^{p}$ such that

$$
\mathfrak{g} \cong \mathfrak{g}_{T_{1}} \oplus \mathfrak{g}^{\prime}
$$

where $T_{1}=\left(e_{1}, 0, \ldots, 0\right) \in V_{n-1, p}^{n-1}$.

Proof. Because $\mathfrak{g} \in \mathcal{F}_{n, m}^{p, 1}$, there is a structure tuple $T=\left(e_{1}, \sum_{j=1}^{n-1} t_{2 j} e_{j}, \ldots, \sum_{j=1}^{n-1} t_{m j} e_{j}\right.$, $0, \ldots, 0) \in V_{n-1, p}^{n-1}$ such that $\mathfrak{g} \cong \mathfrak{g}_{T}$. We can suppose that $t_{i 1}=0$ for all $i \in\{2, \ldots, m\}$. Otherwise, we define using linearity the isomorphism $f$ such that $f\left(e_{j}\right)=e_{j}$ if $j \neq i$ and $f\left(e_{i}\right)=e_{i}+t_{i 1} e_{1}$. Hence, it is sufficient to consider $\mathfrak{g}^{\prime}=\mathfrak{g}_{T^{\prime}} \in \mathcal{F}_{n-1, m-1}^{p}$, where

$$
T^{\prime}=\left(\sum_{j=1}^{n-2} t_{2(j+1)} e_{j}, \ldots, \sum_{j=1}^{n-2} t_{m(j+1)} e_{j}, 0, \ldots, 0\right) \in V_{n-2, p}^{n-2} .
$$

From Theorem 3, the isomorphism classes of $\mathcal{F}_{n, m}^{p, 1}$ are uniquely determined by those of the three families $\mathcal{F}_{n-1, m-1}^{p, i}$, for $i \in\{1,2,3\}$.

We can also suppose that $t_{i 2}=0$ for all $i \geq 2$. Otherwise, we define using linearity the isomorphism $f$ such that $f\left(e_{i}\right)=e_{i}+t_{i 2} e_{1}$ and $f\left(e_{j}\right)=e_{j}$ if $j \neq i$. We now distinguish the following three cases

- Case 1. $t_{i 1}=0$ for all $i \geq 2$. 
- Case 2. $t_{21} \neq 0$.

- Case 3. $t_{21}=0$ and there exists $i>2$ such that $t_{i 1} \neq 0$.

\section{Relationship between isotopism and isomorphism classes of $\mathcal{F}_{n, m}^{p}$.}

The distribution of Lie algebras of $\mathcal{F}_{n}^{p}$ into isotopism classes can be used to determine their distribution into isomorphism classes. Because two isomorphism classes must belong to the same isotopism class, the methodology that we are going to follow to obtain such a distribution is to consider the isotopism class associated to a non-abelian Lie algebra $\mathfrak{g}_{T_{m}} \in \mathcal{F}_{n}^{p}$, for some $m<n$, where $T_{m}=\left(e_{1}, \ldots, e_{m}, 0, \ldots, 0\right) \in V_{n-1, p}^{n-1}$, and to determine all their related isomorphism classes. Let $\mathfrak{g}_{T} \in \mathcal{F}_{n, m}^{p}$, where $T=\left(e_{j_{1}}, \sum_{j=1}^{n-1} t_{2 j} e_{j}, \ldots, \sum_{j=1}^{n-1} t_{m j} e_{j}, 0, \ldots\right.$, $0) \in V_{n-1, p}^{n-1}$ and $j_{1} \in\{1,2, m+1\}$.

Let $(f, f, h)$ be an isotopism between $\mathfrak{g}_{T}$ and $\mathfrak{g}_{T_{m}}$, that is,

$$
[f(u), f(v)]_{\mathfrak{g}_{T m}}=h\left([u, v]_{\mathfrak{g}_{T}}\right) \text {, for all } u, v \in \mathfrak{g}_{T} .
$$

Let $F=\left(f_{i j}\right)$ and $H=\left(h_{i j}\right)$ be the non-singular matrices of order $n-1$ related respectively to $f$ and $h$, that is, such that $f\left(e_{j}\right)=\sum_{i=1}^{n} f_{i j} e_{i}$ and $h\left(e_{j}\right)=\sum_{i=1}^{n} h_{i j} e_{i}$ for all $j \in$ $\{1, \ldots, n\}$. Because $\mathfrak{g}_{T}$ is a Lie algebra of $\mathcal{F}_{n}^{p}$, it is $\left[\mathfrak{g}_{T}, \mathfrak{g}_{T}\right]_{\mathfrak{g}_{T}} \subseteq V_{n-1, p}$ and thus, the numbers $h_{\text {in }}$ do not have any influence in the expression (3), for any $i \in\{1, \ldots, n\}$. The numbers $t_{i j}, f_{i j}$ and $h_{i j}$ are related by the expressions that appear in the following result

Lemma 7. That the following are verified

i. $f_{k i} f_{n j}=f_{n i} f_{k j}$ for all $i, j<n$ and $k \leq m$.

ii. Given $i \leq m$, we have that $\sum_{j=1}^{n-1} t_{i j} h_{k j}=\left\{\begin{array}{l}f_{k i} f_{n n}-f_{n i} f_{k n}, \text { if } k \leq m, \\ 0, \text { otherwise. }\end{array}\right.$

iii. $f_{k i}=f_{n i}=0$ for all $i \in\{m+1, \ldots, n-1\}$ and $k \leq m$.

Proof. The first assertion follows from the fact that, given $i, j<n$, we have

$$
\begin{gathered}
0=h\left(\left[e_{i}, e_{j}\right]_{\mathfrak{g}_{T}}\right)=\left[f\left(e_{i}\right), f\left(e_{j}\right)\right]_{\mathfrak{g}_{T_{m}}}=\left[\sum_{k=1}^{n} f_{k i} e_{k}, \sum_{k=1}^{n} f_{k j} e_{k}\right]_{\mathfrak{g}_{T_{m}}}= \\
=\sum_{k=1}^{m}\left(f_{k i} f_{n j}-f_{n i} f_{k j}\right) e_{k} .
\end{gathered}
$$

Now, given $i<n$, we have

$$
\sum_{k=1}^{n} \sum_{j=1}^{n-1} t_{i j} h_{k j} e_{k}=\sum_{j=1}^{n-1} t_{i j} h\left(e_{j}\right)=h\left(\left[e_{i}, e_{n}\right]_{\mathfrak{g}_{T}}\right)=\left[f\left(e_{i}\right), f\left(e_{n}\right)\right]_{\mathfrak{g}_{T_{m}}}=
$$




$$
=\left[\sum_{k=1}^{n} f_{k i} e_{k}, \sum_{k=1}^{n} f_{k n} e_{k}\right]_{\mathfrak{g}_{T m}}=\sum_{k=1}^{m}\left(f_{k i} f_{n n}-f_{n i} f_{k n}\right) e_{k} .
$$

If $i \leq m$, then the second assertion follows from the coefficients of each $e_{k}$ in the previous expression. Otherwise, because $t_{i j}=0$ for all $i>m$ and $j<n$, we have

$$
f_{k i} f_{n n}=f_{n i} f_{k n}, \text { for all } i>m, j<n \text { and } k \leq m \text {. }
$$

If there exists $i_{0}<n$ such that $f_{n i_{0}} \neq 0$, then (i) implies that there exists a number $\alpha_{k} \in \mathbb{F}_{p}$ such that $f_{k i}=\alpha_{k} f_{n i}$ for all $i<n$. If $i_{0}>m$, then (4) implies that $f_{k n}=\alpha_{k} f_{n n}$ and hence $F$ is non-singular, which is a contradiction. Therefore, $f_{n i}=0$ for all $i \in\{m+1, \ldots, n-1\}$. Now, if $i_{0} \leq m$, then $f_{k i}=\alpha_{k} f_{n i}=0$ for all $k \leq m$ and $i>m$. Finally, if $f_{n i}=0$ for all $i \leq m$, then $f_{n n} \neq 0$ because $F$ is non-singular and thus, (4) implies again that $f_{k i}=0$ for all $k \leq m$ and $i>m$.

Let $M_{T}=\left(m_{i j}\right)$ be the matrix of order $(n-1) \times n$ such that $m_{i j}=t_{i j}$ if $j<n$; otherwise 0 . According to Lemma 7 (ii), it is verified that

$$
M_{T} \cdot H^{t}=\left(\begin{array}{cccccc}
f_{11} f_{n n}-f_{n 1} f_{1 n} & \ldots & f_{m 1} f_{n n}-f_{n 1} f_{m n} & 0 & \ldots & 0 \\
\vdots & \ddots & \vdots & \vdots & \vdots & \vdots \\
f_{1 m} f_{n n}-f_{n m} f_{1 n} & \ldots & f_{m m} f_{n n}-f_{n m} f_{m n} & 0 & \ldots & 0 \\
0 & \ldots & 0 & 0 & \ldots & 0 \\
\vdots & \vdots & \vdots & \vdots & \vdots & \vdots \\
0 & \ldots & 0 & 0 & \ldots & 0
\end{array}\right),
$$

where $H^{t}$ is the transpose of the matrix $H$. Because $H$ is non-singular, we can define the matrix $B=\left(b_{i j}\right)=\left(H^{t}\right)^{-1}$ and thus, it is verified that

$$
t_{i j}=\left\{\begin{array}{l}
\sum_{k=1}^{m}\left(f_{k i} f_{n n}-f_{n i} f_{k n}\right) b_{k j}, \text { for all } i \leq m \text { and } j<n, \\
0, \text { otherwise. }
\end{array}\right.
$$

According to the previous results, the following proposition holds

Proposition 4. An algebra $\mathfrak{g}_{T} \in \mathcal{F}_{n}^{p}$ of structure tuple $T=\left(\sum_{j=1}^{n-1} t_{1 j} e_{j}, \ldots, \sum_{j=1}^{n-1} t_{m j} e_{j}\right.$, $0, \ldots, 0) \in V_{n-1, p}^{n-1}$ is isotopic to $\mathfrak{g}_{T_{m}}$ if and only if there exist two non-singular square matrices of order $n, A=\left(a_{i j}\right)$ and $B=\left(b_{i j}\right)$, such that

i. $a_{k i} a_{n j}=a_{n i} a_{k j}$ for all $i, j<n$ and $k \leq m$.

ii. $a_{k i}=a_{n i}=0$ for all $i \in\{m+1, \ldots, n-1\}$ and $k \leq m$.

iii. $t_{i j}=\sum_{k=1}^{m}\left(a_{k i} a_{n n}-a_{n i} a_{k n}\right) b_{k j}$ for all $i \leq m$ and $j<n$.

From now on, given two non-singular square matrices $A=\left(a_{i j}\right)$ and $B=\left(b_{i j}\right)$ of order $n$ such that $A$ satisfies Conditions (i) and (ii) of Proposition 4, we will denote by $\mathfrak{g}_{A, B}$ the Lie algebra of $\mathcal{F}_{n}^{p}$ whose structure tuple satisfies Condition (iii) of such a result. There are two cases to study 
- Case 1. $a_{n j}=0$ for all $j<n$.

In such a case, Condition (i) is immediately satisfied. Because $A$ is non-singular, it must be true that $a_{n n} \neq 0$ and

$$
t_{i j}=\left\{\begin{array}{l}
a_{n n} \sum_{k=1}^{m} a_{k i} b_{k j}, \text { for all } i \leq m \text { and } j<n, \\
0, \text { otherwise. }
\end{array}\right.
$$

We can suppose that $a_{n n}=1$. Otherwise, it is sufficient to define using linearity the isomorphism $f$ such that $f\left(e_{i}\right)=a_{n n}^{p-1} e_{i}$ for all $i<n$ and $f\left(e_{n}\right)=a_{n n} e_{n}$.

- Case 2. There exists $j_{0} \leq m$ such that $a_{n j_{0}} \neq 0$.

In such a case, from Condition (i) of Proposition 4, given $k \leq m$, there exists $\alpha_{k} \in \mathbb{F}_{n}^{p}$ such that $a_{k j}=\alpha a_{n j}$ for all $j<n$. Hence,

$$
t_{i j}=\left\{\begin{array}{l}
a_{n i} \sum_{k=1}^{m}\left(\alpha_{k} a_{n n}-a_{k n}\right) b_{k j}, \text { for all } i \leq m \text { and } j<n, \\
0, \text { otherwise. }
\end{array}\right.
$$

Observe that, because $A$ is non-singular, it must be true that $a_{k n} \neq \alpha_{k} a_{n n}$ for all $k \leq m$.

If $m=1$, then the previous two cases are reduced to the following

- Case 1.

$$
t_{i j}=\left\{\begin{array}{l}
a_{11} b_{1 j}, \text { if } i=1 \text { and } j<n \\
0, \text { otherwise }
\end{array}\right.
$$

- Case 2.

$$
t_{i j}=\left\{\begin{array}{l}
a_{n 1}\left(\alpha_{1} a_{n n}-a_{1 n}\right) b_{1 j}, \text { if } i=1 \text { and } j<n, \\
0, \text { otherwise. }
\end{array}\right.
$$

We show in Table 3 the isomorphism classes of $\mathcal{F}_{5}^{3}$. 


\begin{tabular}{|c|c|c|}
\hline $\begin{array}{l}\mathfrak{g}_{5}^{1}:(0,0,0,0) \\
\mathfrak{g}_{5}^{4}:\left(0,0, e_{4}, e_{3}\right) \\
\mathfrak{g}_{5}^{7}:\left(0,0,2 e_{4}, e_{3}+e_{4}\right) \\
\mathfrak{g}_{5}^{10}:\left(0,0, e_{3}, e_{4}\right) \\
\mathfrak{g}_{5}^{13}:\left(0, e_{4}, e_{3}, e_{2}+2 e_{4}\right) \\
\mathfrak{g}_{5}^{16}:\left(0,2 e_{4}, e_{3}, e_{2}+2 e_{4}\right) \\
\mathfrak{g}_{5}^{19}:\left(e_{4}, 0, e_{3}, e_{2}+e_{4}\right) \\
\mathfrak{g}_{5}^{22}:\left(0, e_{3}, e_{4}, e_{2}+2 e_{4}\right) \\
\mathfrak{g}_{5}^{25}:\left(0, e_{3}+2 e_{4}, e_{4}, e_{2}+2 e_{4}\right) \\
\mathfrak{g}_{5}^{28}:\left(e_{4}, e_{3}+2 e_{4}, 0, e_{2}\right) \\
\mathfrak{g}_{5}^{31}:\left(e_{4}, e_{3}, e_{2}, e_{1}\right) \\
\mathfrak{g}_{5}^{34}:\left(e_{4}, e_{3}+e_{4}, e_{2}+e_{4}, e_{1}\right) \\
\mathfrak{g}_{5}^{37}:\left(2 e_{4}, e_{3}, e_{2}+e_{4}, e_{1}+e_{4}\right) \\
\mathfrak{g}_{5}^{40}:\left(e_{4}, e_{3}, e_{2}+e_{3}+e_{4}, e_{1}+e_{4}\right) \\
\mathfrak{g}_{5}^{43}:\left(2 e_{4}, e_{3}, e_{2}+e_{3}, e_{1}+2 e_{4}\right) \\
\mathfrak{g}_{5}^{46}:\left(2 e_{4}, 2 e_{3}, e_{2}+e_{4}, e_{1}\right) \\
\mathfrak{g}_{5}^{49}:\left(2 e_{4}, 2 e_{3}+e_{4}, e_{2}+e_{3}+e_{4}, e_{1}+e_{4}\right) \\
\mathfrak{g}_{5}^{52}:\left(e_{3}, 2 e_{4}, e_{2}, e_{1}+e_{4}\right) \\
\mathfrak{g}_{5}^{55}:\left(e_{3}+e_{4}, e_{4}, e_{2}, e_{1}\right) \\
\mathfrak{g}_{5}^{58}:\left(e_{3}+e_{4}, e_{4}, e_{2}+e_{4}, e_{1}+2 e_{4}\right) \\
\mathfrak{g}_{5}^{61}:\left(e_{3}+2 e_{4}, e_{4}, e_{2}, e_{1}\right) \\
\mathfrak{g}_{5}^{64}:\left(e_{3}+2 e_{4}, e_{4}, e_{2}+e_{4}, e_{1}+2 e_{4}\right) \\
\mathfrak{g}_{5}^{67}:\left(e_{3}+2 e_{4}, 2 e_{4}, e_{2}+e_{4}, e_{1}+e_{4}\right) \\
\mathfrak{g}_{5}^{70}:\left(e_{4}, e_{2}, e_{3}, e_{1}+2 e_{4}\right) \\
\mathfrak{g}_{5}^{73}:\left(e_{1}, e_{2}, e_{3}, e_{4}\right) \\
\end{array}$ & $\begin{array}{l}\mathfrak{g}_{5}^{2}:\left(0,0,0, e_{4}\right) \\
g_{5}^{5}:\left(0,0, e_{4}, e_{3}+e_{4}\right) \\
g_{5}^{8}:\left(0, e_{4}, 0, e_{3}\right) \\
\mathfrak{g}_{5}^{11}:\left(e_{3}, 0,0, e_{2}\right) \\
\mathfrak{g}_{5}^{14}:\left(0, e_{4}, e_{3}+e_{4}, e_{2}\right) \\
\mathfrak{g}_{5}^{17}:\left(0,2 e_{4}, e_{3}+e_{4}, e_{2}+2 e_{4}\right) \\
\mathfrak{g}_{5}^{20}:\left(e_{4}, 0, e_{3}, e_{2}+2 e_{4}\right) \\
\mathfrak{g}_{5}^{23}:\left(0, e_{3}+e_{4}, e_{4}, e_{2}\right) \\
\mathfrak{g}_{5}^{26}:\left(e_{4}, e_{3}, 0, e_{2}\right) \\
\mathfrak{g}_{5}^{29}:\left(0, e_{4}, e_{3}, e_{2}\right) \\
\mathfrak{g}_{5}^{32}:\left(e_{4}, e_{3}, e_{2}, e_{1}+e_{4}\right) \\
\mathfrak{g}_{5}^{35}:\left(2 e_{4}, e_{3}, e_{2}, e_{1}\right) \\
\mathfrak{g}_{5}^{38}:\left(e_{4}, e_{3}, e_{2}+e_{3}, e_{1}+e_{4}\right) \\
\mathfrak{g}_{5}^{41}:\left(2 e_{4}, e_{3}, e_{2}+e_{3}, e_{1}\right) \\
\mathfrak{g}_{5}^{44}:\left(2 e_{4}, 2 e_{3}, e_{2}, e_{1}\right) \\
g_{5}^{47}:\left(2 e_{4}, 2 e_{3}, e_{2}+e_{3}, e_{1}+e_{4}\right) \\
\mathfrak{g}_{5}^{50}:\left(e_{3}, e_{4}, e_{2}, e_{1}+e_{4}\right) \\
\mathfrak{g}_{5}^{53}:\left(e_{3}, 2 e_{4}, e_{2}+e_{4}, e_{1}\right) \\
\mathfrak{g}_{5}^{56}:\left(e_{3}+e_{4}, e_{4}, e_{2}, e_{1}+e_{4}\right) \\
\mathfrak{g}_{5}^{59}:\left(e_{3}+e_{4}, 2 e_{4}, e_{2}, e_{1}+e_{4}\right) \\
\mathfrak{g}_{5}^{62}:\left(e_{3}+2 e_{4}, e_{4}, e_{2}+e_{4}, e_{1}\right) \\
\mathfrak{g}_{5}^{65}:\left(e_{3}+2 e_{4}, 2 e_{4}, e_{2}, e_{1}+e_{4}\right) \\
\mathfrak{g}_{5}^{68}:\left(e_{4}, e_{2}, e_{3}, e_{1}\right) \\
\mathfrak{g}_{5}^{71}:\left(2 e_{4}, e_{2}, e_{3}, e_{1}\right)\end{array}$ & $\begin{array}{l}\mathfrak{g}_{5}^{3}:\left(0,0, e_{4}, 0\right) \\
g_{5}^{6}:\left(0,0,2 e_{4}, e_{3}\right) \\
g_{5}^{9}:\left(0, e_{4}, 0, e_{3}+e_{4}\right) \\
\mathfrak{g}_{5}^{12}:\left(0, e_{4}, e_{3}, e_{2}+e_{4}\right) \\
\mathfrak{g}_{5}^{15}:\left(0,2 e_{4}, e_{3}, e_{2}\right) \\
\mathfrak{g}_{5}^{18}:\left(e_{4}, 0, e_{3}, e_{2}\right) \\
\mathfrak{g}_{5}^{21}:\left(e_{4}, 0, e_{3}+e_{4}, e_{2}+e_{4}\right) \\
\mathfrak{g}_{5}^{24}:\left(0, e_{3}+e_{4}, e_{4}, e_{2}+e_{4}\right) \\
\mathfrak{g}_{5}^{27}:\left(e_{4}, e_{3}+e_{4}, 0, e_{2}+e_{4}\right) \\
\mathfrak{g}_{5}^{30}:\left(0, e_{2}, e_{3}, e_{4}\right) \\
\mathfrak{g}_{5}^{33}:\left(e_{4}, e_{3}, e_{2}+e_{4}, e_{1}\right) \\
\mathfrak{g}_{5}^{36}:\left(2 e_{4}, e_{3}, e_{2}, e_{1}+e_{4}\right) \\
\mathfrak{g}_{5}^{39}:\left(e_{4}, e_{3}, e_{2}+e_{3}, e_{1}+2 e_{4}\right) \\
\mathfrak{g}_{5}^{42}:\left(2 e_{4}, e_{3}, e_{2}+e_{3}, e_{1}+e_{4}\right) \\
g_{5}^{45}:\left(2 e_{4}, 2 e_{3}, e_{2}, e_{1}+e_{4}\right) \\
g_{5}^{48}:\left(2 e_{4}, 2 e_{3}, e_{2}+e_{3}+e_{4}, e_{1}+e_{4}\right) \\
g_{5}^{51}:\left(e_{3}, e_{4}, e_{2}+e_{4}, e_{1}\right) \\
\mathfrak{g}_{5}^{54}:\left(e_{3}, 2 e_{4}, e_{2}+e_{4}, e_{1}+2 e_{4}\right) \\
\mathfrak{g}_{5}^{57}:\left(e_{3}+e_{4}, e_{4}, e_{2}+e_{4}, e_{1}+e_{4}\right) \\
\mathfrak{g}_{5}^{60}:\left(e_{3}+e_{4}, 2 e_{4}, e_{2}+e_{4}, e_{1}\right) \\
\mathfrak{g}_{5}^{63}:\left(e_{3}+2 e_{4}, e_{4}, e_{2}+e_{4}, e_{1}+e_{4}\right) \\
\mathfrak{g}_{5}^{66}:\left(e_{3}+2 e_{4}, 2 e_{4}, e_{2}+e_{4}, e_{1}\right) \\
\mathfrak{g}_{5}^{69}:\left(e_{4}, e_{2}, e_{3}, e_{1}+e_{4}\right) \\
\mathfrak{g}_{5}^{72}:\left(2 e_{4}, e_{2}, e_{3}, e_{1}+2 e_{4}\right)\end{array}$ \\
\hline
\end{tabular}

Table 3: Isomorphism classes of $\mathcal{F}_{5}^{3}$.

\begin{tabular}{|c|c|c|c|}
\hline$n$ & morphism classes & & \\
\hline 2 & $f_{2}^{1}:(0)$ & $f_{2}^{2}:\left(e_{1}\right)$ & \\
\hline \multirow[t]{3}{*}{3} & $f_{3}^{1}:(0,0)$ & $f_{3}^{\overline{2}}:\left(0, e_{2}\right)$ & $f_{3}^{3}:\left(e_{2}, 0\right)$ \\
\hline & $f_{3}^{4}:\left(e_{2}, e_{1}\right)$ & $f_{3}^{5}:\left(e_{1}, e_{2}\right)$ & $f_{3}^{6}:\left(e_{2}, e_{1}+e_{2}\right)$ \\
\hline & $\begin{array}{l}f_{3:}^{7}:\left(e_{2}, e_{1}+2 e_{2}\right) \\
f_{3}^{10}:\left(e_{1}, e_{2}\right)\end{array}$ & $f_{3}^{8}:\left(2 e_{2}, e_{1}\right)$ & $f_{3}^{9}:\left(2 e_{2}, e_{1}+2 e_{2}\right)$ \\
\hline 4 & $f_{4}^{1}:(0,0,0)$ & $f_{4}^{2}:\left(0,0, e_{3}\right)$ & $f_{4}^{3}:\left(0, e_{3}, 0\right)$ \\
\hline & $f_{4}^{4}:\left(0, e_{3}, e_{2}\right)$ & $f_{4}^{5}:\left(0, e_{3}, e_{2}+e_{3}\right)$ & $f_{4}^{6}:\left(0, e_{3}, e_{2}+2 e_{3}\right)$ \\
\hline & $f_{4}^{7}:\left(0,2 e_{3}, e_{2}\right)$ & $f_{4}^{8}:\left(0,2 e_{3}, e_{2}+e_{3}\right)$ & $f_{4}^{9}:\left(0,2 e_{3}, e_{2}+2 e_{3}\right)$ \\
\hline & $\begin{array}{l}f_{4}^{10}:\left(e_{3}, 0, e_{2}\right) \\
f_{4}^{13}:\left(e_{3}, e_{2}, e_{1}\right)\end{array}$ & $\begin{array}{l}f_{4}^{11}:\left(e_{3}, 0, e_{2}+e_{3}\right) \\
f_{4}^{14}:\left(e_{3}, e_{2}, e_{1}+e_{3}\right)\end{array}$ & $\begin{array}{l}f_{4}^{12}:\left(0, e_{2}, e_{3}\right) \\
f_{4}^{15}:\left(e_{3}, e_{2}, e_{1}+2 e_{3}\right)\end{array}$ \\
\hline & $f_{4}^{16}:\left(e_{3}, e_{2}, e_{1}+3 e_{3}\right)$ & $f_{4}^{17}:\left(e_{3}, e_{2}, e_{1}+4 e_{3}\right)$ & $f_{4}^{18}:\left(e_{3}, e_{2}+e_{3}, e_{1}\right)$ \\
\hline & $f_{4}^{19}:\left(2 e_{3}, e_{2}, e_{1}\right)$ & $f_{4}^{20}:\left(2 e_{3}, e_{2}, e_{1}+e_{3}\right)$ & $f_{4}^{21}:\left(2 e_{3}, e_{2}, e_{1}+2 e_{3}\right)$ \\
\hline & $f_{45}^{22}:\left(2 e_{3}, e_{2}, e_{1}+3 e_{3}\right)$ & $f_{4}^{23}:\left(2 e_{3}, e_{2}, e_{1}+4 e_{3}\right)$ & $f_{4}^{24}:\left(3 e_{3}, e_{2}, e_{1}\right)$ \\
\hline & $f_{48}^{\overline{2} 5}:\left(3 e_{3}, e_{2}, e_{1}+e_{3}\right)$ & $f_{4}^{26}:\left(3 e_{3}, e_{2}, e_{1}+3 e_{3}\right)$ & $f_{4}^{27}:\left(3 e_{3}, e_{2}, e_{1}+4 e_{3}\right)$ \\
\hline & $f_{4}^{2} 8:\left(4 e_{3}, e_{2}, e_{1}+e_{3}\right)$ & $f_{4}^{29}:\left(4 e_{3}, e_{2}, e_{1}+2 e_{3}\right)$ & $f_{4}^{30}:\left(4 e_{3}, e_{2}, e_{1}+4 e_{3}\right)$ \\
\hline & $f_{4}^{31}:\left(4 e_{3}, e_{2}+e_{3}, e_{1}+2 e_{3}\right)$ & $f_{45}^{32}:\left(e_{2}, e_{3}, e_{1}+e_{3}\right)$ & $f_{4}^{33}:\left(e_{2}, e_{3}, e_{1}+2 e_{3}\right)$ \\
\hline & $f_{4}^{34}:\left(e_{2}+e_{3}, e_{3}, e_{1}+e_{3}\right)$ & $f_{4}^{35}:\left(e_{2}+e_{3}, e_{3}, e_{1}+3 e_{3}\right)$ & $f_{49}^{36}:\left(e_{2}+e_{3}, 2 e_{3}, e_{1}\right)$ \\
\hline & $f_{4}^{37}:\left(e_{2}+e_{3}, 2 e_{3}, e_{1}+2 e_{3}\right)$ & $\begin{array}{l}f_{4}^{38}:\left(e_{2}+e_{3}, 2 e_{3}, e_{1}+4 e_{3}\right) \\
f^{41}:\left(e_{2}+2 e_{3}, 2 e_{3}, e_{1}\right)\end{array}$ & $f_{4}^{39}:\left(e_{2}+2 e_{3}, e_{3}, e_{1}+e_{3}\right)$ \\
\hline & $f_{4}:\left(e_{2}+2 e_{3}, e_{3}, e_{1}\right.$ & $\left(e_{2}+2 e_{3}, 2 e_{3}, e_{1}\right)$ & $:\left(e_{1}, e_{2}, e_{3}\right)$ \\
\hline
\end{tabular}

Table 4: Isomorphism classes of $\mathcal{F}_{n}^{5}$, for $2 \leq n \leq 4$.

\section{References}

[1] A. A. Albert, Non-Associative Algebras: I. Fundamental Concepts and Isotopy, Annals of Mathematics, Second Series, 43:4 (1942), 685-707.

[2] M. Babikov, Isotopy and identities in alternative algebras, Proc. Am. Math. Soc. 125:6 (1997), 1571-1575.

[3] L. Boza, E. M. Fedriani and J. Núñez, Una relación entre los pseudografos dirigidos sin aristas repetidas y algunas álgebras de Lie. Proceedings of IV Encuentro Andaluz de Matemática Discreta (2005), 99-104. 
[4] L. Boza, E. M. Fedriani, J. Núñez, A. M. Pacheco and M. T. Villar, Directed pseudographs and Lie algebras over finite fields, Czech. Math. J. 64(139), 229-239 (2014).

[5] O. J. Falcón, R. M. Falcón and J. Núñez, Isomorphism and isotopism classes of filiform Lie algebras of dimension up to seven. Avalaible from http://arxiv.org/pdf/1510.07066v1.pdf.

[6] E. Falconer, Quasi-group identities invariant under isotopy, Ph.D. thesis, Emory University (1969).

[7] J. Hora, Autotopisms and isotopisms of trilinear alternating forms, Comm. Algebra 40:4 (2012), 1438-1455.

[8] B. D. McKay, A. Meynert and W. Myrvold, Small Latin Squares, Quasigroups and Loops, J. Combin. Des. 15:2 (2007), 98-119.

[9] J. Núñez, A. Pacheco and M. T. Villar, Study of a family of Lie algebras over $\mathbb{Z} / 3 \mathbb{Z}$. International Journal of Applied Mathematics and Statistics, Special volume 7:W10 (2010), 40-45.

[10] H. P. Petersson, Isotopisms of Jordan algebras, Proc. Amer. Math. Soc. 20 (1969), 477-482.

[11] S. J. Thomas Schwarz, Small non-associative division algebras up to isotopy, Algebra Discrete Math. 9:1 (2010), 103-108. 